\title{
Optimization of Fin with Rectangular and Triangular Shapes by Levenberg - Marquardt Method
}

\author{
Long Nhut-Phi Nguyen ${ }^{1}$, Quan Nguyen ${ }^{2 *}$, Son Hoai Nguyen ${ }^{3}$ \\ ${ }^{1}$ Faculty of Mechanical Engineering, HCMC University of Technology and Education (HCMUTE), Thu Duc 71300, Ho Chi \\ Minh City, Viet Nam \\ ${ }^{2}$ Faculty of Engineering - Technology, Pham Van Dong University (PDU), Quang Ngai 53100, Quang Ngai Province, Viet \\ Nam \\ ${ }^{3}$ Faculty of Civil Engineering, HCMC University of Technology and Education (HCMUTE), Thu Duc 71300, Ho Chi Minh \\ City, Viet Nam
}

Corresponding Author Email: nquan@ @du.edu.vn

https://doi.org/10.18280/mmep.090130

Received: 27 July 2021

Accepted: 28 December 2021

\section{Keywords:}

Levenberg - Marquardt method, rectangular fin, triangular fin, shape optimization

\section{INTRODUCTION}

The fin plays an essential role in cooling devices such as automobile engines, electronic devices, air condition systems, ambient air, etc. In recent decades, fin optimization addressed to help create a compact design and reduce production costs [1]. Thus, many researchers still focused on the optimal problems for fin.

When the rate of heat transfer is known, the minimum volume of the fins is a popular parameter to be optimized. For the first time, the fin profile is parabolic, which has been investigated by Schmidt [2]. The evaluation of the results by performing some strong mathematical models [3] and the results in the study [4] have proved that the problem in Ref. [2] is still valid for the optimization of the fin in the case where the convection heat transfer coefficient varies according to the power law. The simpler approach for the fin optimizing problem to achieve the maximum possible heat transfer rate in a defined volume unit or to meet the heat dissipation capacity with a minimum volume is to select the right profile for the fin (e.g., triangular and/or rectangular profile, etc.).

However, in optimizing the fin with the parabolic profile, the curved surface with the thickness at the top is almost zero, increasing complexity and high cost in actual production. It is easier and now widely used to design and fabricate fins for heat dissipation systems with triangular and/or rectangular profiles, allowing for a quicker approach. Therefore, the optimization of fin size with triangular and/or rectangular profiles has been presented in many studies. Assuming constant heat parameters, no significant influence of initial heat transfer, the approximation for the one-way heat transfer equation: the common profile of the fin has been optimized in
[1]. An overview of the optimization of fin size with common profiles has been presented by Aziz [5]. The optimization problem with convection boundary conditions for triangular and rectangular radiator fins has been studied [6]. The optimum design has been performed with step-change according to the cross-sectional area of the rectangular fins according to the constant thermal parameter [7]. Similarly, the results for optimizing the rectangular profile of the fin provide many ideas for subsequent studies [8-11]. Most of the above studies performed the optimization based on analytical methods, not yet solving general nonlinear problems in the fin design. At the same time, not yet much-published research proposes an effective method in the design optimization of the size of the fins with triangular and/or rectangular profiles in the linear and/or rectangular case nonlinear.

Through slanting perforation for rectangular fins to improve efficiency, the Degenerate Hypergeometric Equation (DH Equation) was used to find the energy and the Signum function used to model the heat transfer zone. The two inputs: angle and size of perforations of the existing model, have been optimized to achieve the largest heat transfer area and the smallest fin weight. However, the optimal score results are outside the range of inclination angles mentioned in the study [12].

The research on optimizing rectangular fin design in natural convection has been carried out by analyzing heat transfer characteristics and flow patterns in the fin structure. The CFD (Computational Fluid Dynamics) optimization algorithm determined the impact on heat transfer through the parameters of fin spacing, fin length, and height. By dynamic-Q algorithm, the results of the optimal global design show that the above parameters strongly influence the flow through the ends of the fin channel [13]. 
The heat exchanger structure with the wavy fin-andelliptical tube has been optimized with a combination of Latin Hypercube (LH) sampling, Computational Fluid Dynamics (CFD) simulation, and the radical basis function. Optimal parameters include the Colburn coefficient, the friction factor, air temperature, pressure, heat transfer rate. The results showed that the Colburn coefficient increased by about $5 \%$, the coefficient of friction decreased by about $23 \%$, the air temperature raised by $4.6 \mathrm{~K}$, the pressure decreased by $10 \%$, and the heat transfer rate was faster. At the same time, the use of the field synergy principle to explain the optimal heat transfer and efficiency basis is the theoretical basis for optimizing the design of the heat exchanger structure [14].

Besides, the study used the modified Newton - Raphson (MNR) method to optimize the volume of a longitudinal fin with a few typical profiles, showing high efficiency $[15,16]$. However, the MNR method has some disadvantages, such as the convergence problem may fail if choosing an inappropriate initial value, especially in highly non-linear problems. Therefore, it is necessary to propose a stable and simple solution to achieve the best convergence rate.

In this article, the Levenberg - Marquardt (L - M) method is proposed to solve the optimal design problem: optimizing the size of the longitudinal fin to achieve the minimum volume. This method is shown to be effective in applying to non-linear problems, such as the optimization problem performed in Ref. [16]. The "volume updating" mechanism has been inset to the $\mathrm{L}$ - $\mathrm{M}$ algorithm to solve the optimization problem and is implemented as the inverse problem. Specifically, the finite element method (FEM) solves the heat transfer process of the fin (forward problem), and the optimal volume of the fin is found by the L - M method (inverse problem). In the following section, this article presents in detail showing the high feasibility of the proposed method.

\section{PROBLEM STATEMENT}

Figure 1 shows the two-dimensional and three-dimensional triangular (left) and rectangular (right) profiles of the longitudinal fin.
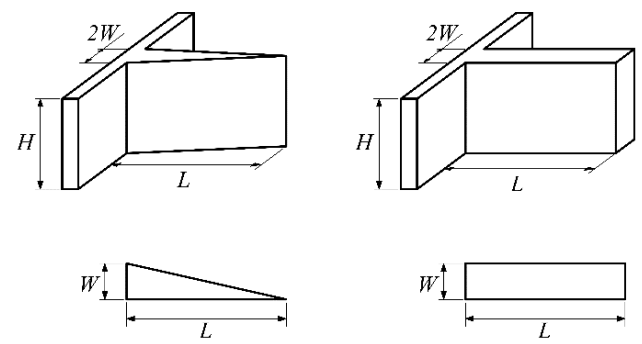

Figure 1. The triangular and rectangular longitudinal fin

The heat transfer equation for a fin with a two-dimensional profile, cooled by natural convection and radiation, in a steady-state, without an internal heat source, is shown as following [16]:

$$
\frac{\partial}{\partial x}\left(k \frac{\partial \theta}{\partial x}\right)+\frac{\partial}{\partial y}\left(k \frac{\partial \theta}{\partial y}\right)=0 \text { in the fin domain }
$$

and the boundary conditions are expressed as follows:

$$
\begin{gathered}
-k A_{b} \frac{\partial \theta}{\partial x}=q_{i n} \text { at the fin base } \\
-k\left(\frac{\partial \theta}{\partial x}+\frac{\partial \theta}{\partial y}\right) \cdot \mathbf{n}=h\left(\theta-\theta_{\infty}\right)+\varepsilon \sigma\left(\theta^{4}-\theta_{\text {sur }}^{4}\right) \text { at the } \\
\text { convective surface } \\
k \frac{\partial \theta}{\partial y}=0 \text { at the fin symmetric }
\end{gathered}
$$

where, $\theta$ is the undefined temperature field in the fin domain, $k$ is the thermal conductivity, $A_{\mathrm{b}}$ is the cross-sectional area at the fin base, $q_{\text {in }}$ is the total of toward the inside heat loss at the base, $h$ is the convective heat transfer coefficient, $\varepsilon$ is the emissivity coefficient, $\sigma$ is Stefan-Boltzmann constant, $\theta_{\infty}$ is the ambient temperature, $\theta_{\text {sur }}$ is the surrounding environment temperature, $\mathbf{n}$ is the convective surface exterior normal vector.

The finite element method is used to solve the forward problem: with the fin dimensions and boundary conditions known, the fin and the base's temperature field results are achieved [17].

\section{METHODOLOGY}

\subsection{Levenberg - Marquardt method (L - M method)}

The dimensions of the fin are described by length (L) and width $(2 \mathrm{~W})$ for both triangular and rectangular profiles (see Figure 1). The optimization results in this article: the longitudinal fin volume is minimized so that the heat loss is given and the base temperature is specified. Thus, the posed problem included the two optimal variables: 1 - length and w width of the fin (see Figure 2).

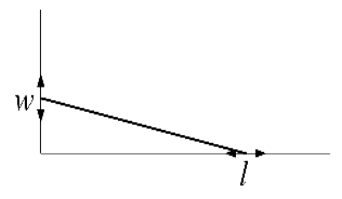

(a) Triangular fin

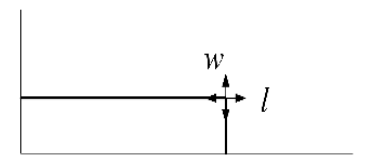

(b) Rectangular fin
Figure 2. The optimal variables for two profiles

To ensure continuity in the optimization process, the control points' positions satisfy Eq. (5):

$$
\left\{\begin{array}{l}
w>0 \\
l>0
\end{array}\right.
$$

The application of the L - M method to specify the optimal value for the variables needs to provide the desired base temperature $\theta_{e}$ and the desired fin volume $V_{e}$. Firstly, through the forward problem, the calculated temperature $\theta_{c}^{i}$ and the calculated volume $V_{c}$ are recorded. Next, the optimal dimension estimation with minimize volume is done by minimizing the sum of the square equation as follows:

$$
S(w, l)=\sum_{i=1}^{N}\left(\theta_{c}^{i}-\theta_{e}\right)^{2}+\left(\hat{V}_{c}-\theta_{e}\right)^{2}=\sum_{i=1}^{N+1}\left(\Theta_{i}-\theta_{e}\right)^{2}
$$


where, $N$ is the calculated temperature point number at the fin base, $\Theta_{i}$ consist $\theta_{c}^{i}$ and $\widehat{V}_{c}$.

The normalized volume $\widehat{V}_{c}$ is expressed as follow:

$$
\hat{V}_{c}=\frac{V_{c}}{V_{e}} \cdot \theta_{e}
$$

To minimize $S(x, l)$ (in Eq. (6)), the values of the length and width fin, $(x, l)$, fulfill the following set of nonlinear equations:

$$
\sum_{i=1}^{N+1} \frac{\partial \boldsymbol{\Theta}}{\partial \mathbf{x}}\left(\Theta_{i}-\theta_{e}\right)=0
$$

where, $\mathbf{x}=\{w, l\}$ is the vector including the width variable of $w$ and the length variable of $l$.

In other words, the solution of Eq. (8) is the fin optimal width and length value of the fin. In this article, Eq. (8) is solved by the $\mathrm{L}-\mathrm{M}$ method. The iteration of the convergence is controlled through the $\mu$ parameter in the proposed method. The equation to find the optimal variables using the L - M method is written as follows:

$$
\mathbf{x}^{k+1}=\mathbf{x}^{k}-\left(\mathbf{D}^{T} \mathbf{D}+\mu \boldsymbol{\Omega}\right)^{-1} \mathbf{D}^{T}\left(\boldsymbol{\Theta}-\theta_{e}\right)
$$

where, $\boldsymbol{D}=\frac{\partial \boldsymbol{\theta}}{\partial \boldsymbol{x}}$ is the sensitivity matrix, $\boldsymbol{\Omega}$ is the diagonal matrix and $k$ is the iteration index.

The two parameters that need to be given in Eq. (9) for a solution are the desired base temperature and the suitable volume. In which the fin volume is undetermined. It is the parameter that the goal of this study performs optimization.

Based on the "curve fitting" mechanism in the L - M method, a mechanism called "volume updating" is inset in the L - M algorithm to optimize the required parameter. With this mechanism, the finest approximation value, which minimizes the sum of squared differences between the calculated and estimated values, is the result of the solution. The larger the number of the calculated temperature point $\mathrm{N}$, the more accurate the solution obtained. Here are the four basic steps of the "volume updating" mechanism [16]:

Step 1: Set the N value is large enough, and the beginning fin volume value is small.

Step 2: Solve Eq. (6) - Eq. (9) to find the fin volume.

Step 3: Update the new value in Step 2 and return to Step 1.

Step 4: Stop the algorithm when the stopping criteria is pleased.

\subsection{The stopping criteria}

To achieve the goal of the posed problem: the fin volume reaches the minimum value, two optimal variables $w$ and $l$ in the vector $\mathbf{x}$ have been mentioned. In the $\mathrm{L}-\mathrm{M}$ method, the iterative process to find $\mathbf{x}^{k+1}$ from $\mathbf{x}^{k}$ into Eq. (9) stops if please two criteria as follows [18]:

$$
\left\{\begin{array}{l}
\boldsymbol{\Theta}-\theta_{e} \geq 0 \\
\left\|S\left(\mathbf{x}^{k+1}\right)-S\left(\mathbf{x}^{k}\right)\right\| \leq \delta\left\|S\left(\mathbf{x}^{k+1}\right)\right\|
\end{array}\right.
$$

and the stopping criterion of iteration in the $\mathrm{L}-\mathrm{M}$ method is stated:

$$
\left\|\boldsymbol{\Theta}-\theta_{e}\right\| \leq e\left\|\theta_{e}\right\|
$$

or

$$
\left\|S\left(\mathbf{x}^{k+1}\right)-S\left(\mathbf{x}^{k}\right)\right\| \leq \delta\left\|S\left(\mathbf{x}^{k+1}\right)\right\|
$$

where, $e$ and $\delta$ are the convergence tolerances.

\subsection{Computational algorithm}

Set the convergence tolerance $e$ and $\delta$, the beginning control point $\mathbf{x}^{0}$, the beginning fin volume $V_{e}^{0}$, and the adjusting factor $\mu$. At the $k$-th iteration, the $\mathbf{x}^{\mathrm{k}}$ value is known, and the $\mathbf{x}^{k+1}$ value is estimated as follows:

Step 1: Calculate $\theta_{c}$ base on Eq. (1) - Eq. (4) (the forward problem).

Step 2: Compute $\mathbf{x}^{k+1}$ by Eq. (9) and specify the new $\boldsymbol{S}$ through Eq. (6).

Step 3: If $S\left(\boldsymbol{x}^{k+1}\right)>S\left(\boldsymbol{x}^{k}\right)$ or Eq. (5)'s condition is not pleased, reset $\mu^{k}=10 \mu^{k}$, and go back to Step 3. Otherwise, get the new $\boldsymbol{x}^{k+1}$.

Step 4: Update the new fin volume if Eq. (10)'s criterion is pleased, reset $k$ by $k+1, \mu^{k}=0.1 \mu^{k}$ and go back to Step 1 .

Step 5: Finish the algorithm if the stopping criterion in Eq. (11) and Eq. (12) are pleased. Otherwise, reset $k$ by $k+1, \mu^{k}=$ $0.1 \mu^{k}$ and go back to Step 1 .

\section{RESULTS AND DISCUSSIONS}

This section of the research presents two specific cases to find the value for the optimal variables to achieve the minimum longitudinal fin volume with both triangular and rectangular profiles, demonstrating the feasibility of the proposed method. Besides, the results of this optimization problem are also compared with the results (the theory [1]) and the modified Newton - Raphson (MNR) method [16].

\subsection{Case 1}

In case 1 , the optimization problem is carried out for fin with the triangular profile. The initial data of the problem are as follows:

The fin height: $H=0.2 \mathrm{~m}$;

The thermal conductivity: $k=58.3 \frac{\mathrm{W}}{\mathrm{mK}}$;

The desirable dissipates heat flow: $Q=20 \mathrm{~W}$;

The base temperature: $\theta_{b}=400 \mathrm{~K}$;

The surrounding temperature: $\theta_{a}=300 \mathrm{~K}$;

The convective heat transfer: $\bar{h}=5.2564 \frac{W}{m^{2} K}[16]$;

The beginning volume: $V_{e}^{0}=4 e-5 \mathrm{~m}^{3}$;

The beginning dimensions: $1_{0}=0.3 \mathrm{~m}$ and $\mathrm{w}_{0}=0.001 \mathrm{~m}$;

The stopping criteria values: $\delta=10^{-4}$ and $\varepsilon=10^{-4}$.

The optimal results obtained by the theory [1], and the MNR method [16], and the L - M method for the triangular shape are shown in Table 1 .

Additionally, the relative error of the fin optimal parameters between the L - M method with [1] and between the L - M method with the MNR method [16] for the triangular shape (see Table 2). 
Table 1. Compare the optimal results between the L-M method, the theory [1], and the modified Newton - Raphson (MNR) method [16] for the triangular shape

\begin{tabular}{cccc}
\hline $\begin{array}{c}\text { The fin optimal } \\
\text { parameters }\end{array}$ & $\begin{array}{c}\text { The } \mathbf{L}-\mathbf{M} \\
\text { method }\end{array}$ & $\begin{array}{c}\text { The } \\
\text { theory [1] }\end{array}$ & $\begin{array}{c}\text { The MNR } \\
\text { method [16] }\end{array}$ \\
\hline $\begin{array}{c}\text { The } l \text { length } m \\
\text { The } w \text { width } m\end{array}$ & $1.6024 e-1$ & $1.6022 e-1$ & $1.6020 e-1$ \\
$1.3494 e-3$ & $1.3498 e-3$ & $1.3503 e-3$ \\
$\begin{array}{c}\text { The } V \text { minimum } \\
\text { volume } m^{3}\end{array}$ & $4.3246 e-5$ & $4.3255 e-5$ & $4.3265 e-5$ \\
\hline
\end{tabular}

Table 2. The relative error between the $\mathrm{L}$ - $\mathrm{M}$ method with the theory [1] and between the L - M method with the MNR method [16] for the triangular shape

\begin{tabular}{lcc}
\hline \multirow{2}{*}{$\begin{array}{c}\text { The fin optimal } \\
\text { parameters }\end{array}$} & $\begin{array}{c}\text { The } \mathbf{L}-\mathbf{M} \\
\text { method and } \\
{[\mathbf{1}]}\end{array}$ & $\begin{array}{c}\text { The L - M method } \\
\text { and the MNR } \\
\text { method }[\mathbf{1 6}]\end{array}$ \\
\hline $\begin{array}{l}\text { The } l \text { length } \\
\text { The } w \text { width }\end{array}$ & 0.012 & 0.025 \\
The $V$ minimum & 0.030 & 0.067 \\
volume & 0.022 & 0.092 \\
\hline
\end{tabular}

Furthermore, the relation between the temperature and the length in case the triangular shape of the theory [1], the MNR method [16], and the L - M method (the Present) is illustrated in Figure 3.

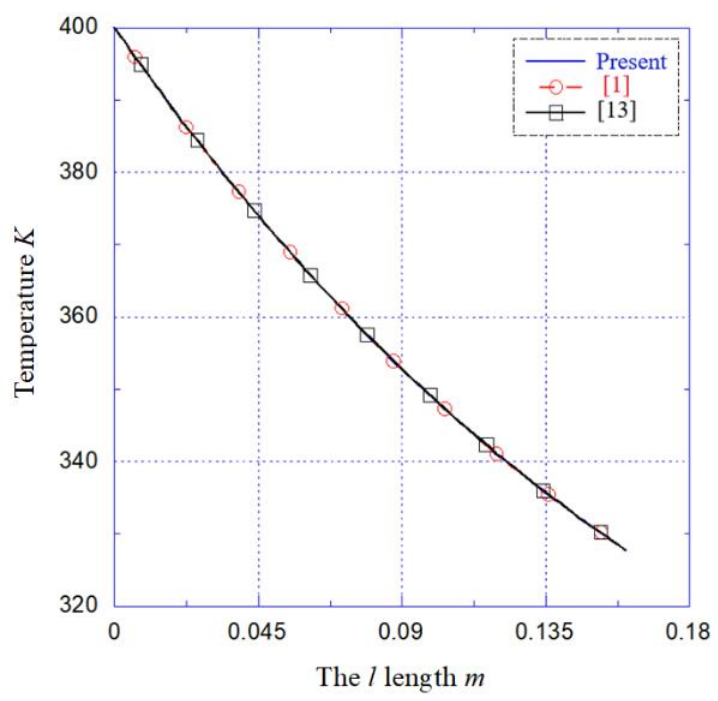

Figure 3. The temperature versus the length in case the triangular shape

As shown, in Case 1, the optimized values of longitudinal fin with the triangular profile: the length of $1=0.16024 \mathrm{~m}$, the width of $w=1.3494 \mathrm{e}-3 \mathrm{~m}$, and the minimum volume $V=$ $4.3246 e-5 \mathrm{~m}^{3}$. Besides, the relative error between the [1], [16] and the Present studies is very small. The largest relative error is $0.092 \%$ for the optimal volume between the L - M method with the MNR method [16] (see Table 2). This means that the L - M method can accurately estimate the minimum volume value of fin with the triangular profile through the desirable dissipate heat flow and the base temperature. Furthermore, the L - M method achieves results after 25 iterations, while the MNR method [16] is more than 100 iterations. This shows that the L - M method (the Present) has the faster convergence rate than the MNR method [16].

\subsection{Case 2}

The optimal fin problem with rectangular shape is investigated. The thermal properties are similar to Case 1 . In case 2 , The beginning dimensions: $l_{0}=0.2 \mathrm{~m}$ and $w_{0}=$ $0.001 \mathrm{~m}$.

The optimal results achieved by the theory [1], and the modified Newton - Raphson (MNR) method [16], and the L $\mathrm{M}$ method for the rectangular shape are visible in Table 3.

Table 3. Compare the optimal results between the L - M method, the theory [1], and the modified Newton - Raphson (MNR) method [16] for the rectangular shape

\begin{tabular}{|c|c|c|c|}
\hline $\begin{array}{c}\text { The fin optimal } \\
\text { parameters }\end{array}$ & $\begin{array}{c}\text { The L-M } \\
\text { method }\end{array}$ & $\begin{array}{c}\text { The theory } \\
{[1]}\end{array}$ & $\begin{array}{c}\text { The MNR } \\
\text { method [16] }\end{array}$ \\
\hline The $l$ length $m$ & $1.5025 e-1$ & $1.5178 e-1$ & $1.5022 e-1$ \\
\hline The $w$ width $m$ & $1.0347 e-3$ & $1.0313 e-3$ & $1.0350 e-3$ \\
\hline $\begin{array}{l}\text { The } V \text { minimum } \\
\text { volume } \mathrm{m}^{3}\end{array}$ & $6.2188 e-5$ & $6.2613 e-5$ & $6.2188 e-5$ \\
\hline
\end{tabular}

The relative error of the fin optimal parameters in Table 4 is between the L - M method with [1] and between the L - M method with the MNR method [16] for the rectangular shape.

Table 4. The relative error between the $\mathrm{L}$ - $\mathrm{M}$ method with [1] and between the L - M method with the MNR method [16] for the rectangular shape

\begin{tabular}{lcc}
\hline \multirow{2}{*}{$\begin{array}{c}\text { The fin optimal } \\
\text { parameters }\end{array}$} & $\begin{array}{c}\text { The L - M } \\
\text { method and } \\
{[\mathbf{1 1}}\end{array}$ & $\begin{array}{c}\text { The L - M method } \\
\text { and the MNR } \\
\text { method [16] }\end{array}$ \\
\hline The $l$ length & 1.01 & 0.02 \\
The $w$ width & 0.33 & 0.03 \\
The $V$ minimum & 0.68 & 0 \\
volume & & 0 \\
\hline
\end{tabular}

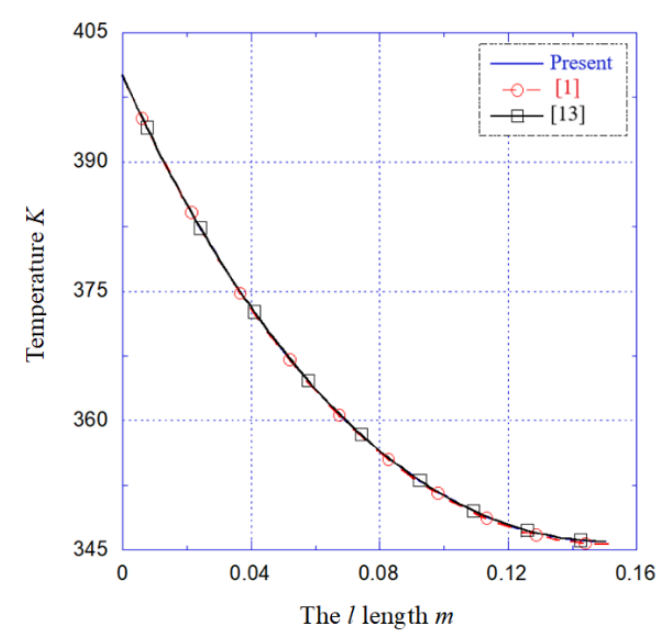

Figure 4. The temperature versus the length in case the rectangular shape

Additionally, the relation between the temperature and the length in case the rectangular shape of the theory [1], the MNR method [16], and the L - M method (the Present) is illustrated in Figure 4.

In Case 2, the optimized values of longitudinal fin with the rectangular profile: the length of $l=0.15025 \mathrm{~m}$, the width of $w=1.0347 e-3 \mathrm{~m}$, and the minimum volume $V=6.2188 e-5 \mathrm{~m}^{3}$. In 
Table 4, the relative error of the optimal volume between the $\mathrm{L}-\mathrm{M}$ method and [1] is $0.68 \%$, while that of between the $\mathrm{L}$ $\mathrm{M}$ method and the MNR method [16] is $0 \%$. Because the forward problem in the L - M method and the MNR method [16] is solved by the FEM method for the two-dimension model with the convective tip, so, the minimum volume value in two methods: the L - M method and the MNR method [16] is smaller. Besides, the results of the problem in [1] applying the one-dimensional model without the convective tip.

Furthermore, the convergence rate by the L - M method (34 iterations) is also dramatically more than that by the MNR method [16] (more 110 iterations). This shows that the L - M method (the Present) has a fast convergence rate, more than the MNR method [16]. Again showing the L - M method in this study is potent and efficient than the MNR method in [16].

\section{CONCLUSION}

This article, optimizing the size of the longitudinal fin, with triangular and rectangular shapes, to achieve the minimum volume, is implemented by the Levenberg - Marquardt (L - M) method. The "volume updating" mechanism is inset to the $\mathrm{L}$ $\mathrm{M}$ algorithm to determine the longitudinal fin volume is minimized so that the heat loss is given and the base temperature is expected. The results in the two given cases, applying the L - M method, are in good agreement with the results in the theory [1] and the MNR method [16]. Moreover, the relative error of the method presented in this article is very tiny with the two compared methods and has a much faster convergence rate than the MNR method [16]. Thereby, it is shown that the $\mathrm{L}-\mathrm{M}$ method is a very effective and reliable method. At the same time, another highlight of this method is that it does not depend on the type of forwarding problem. Therefore, the Levenberg - Marquardt (L - M) method (the Present) contributes to reducing the cost of calculation, fin design, and improving productivity in actual production. Moreover, this method can be applied to the other fin shapes and considered to three-dimensional fin problems.

\section{ACKNOWLEDGMENT}

This work belongs to the project grant No: T2021-25TÐ funded by Ho Chi Minh City University of Technology and Education, Vietnam.

\section{REFERENCES}

[1] Kraus, A.D., Aziz, A., Welty, J. (2007). Extended Surface Heat Transfer. John Wiley. ISBN: 978-0-47017258-2.

[2] Schmidt, E. (1926). Die Wärmeübertragung durch Rippen.

[3] Duffin, R.J. (1959). A variational problem relating to cooling fins. Indiana Univ. Math. J., 8(1): 47-56. https://doi.org/10.1512/iumj.1959.8.58003

[4] Natarajan, U., Shenoy, U.V. (1990). Optimum shapes of convective pin fins with variable heat transfer coefficient. Journal of the Franklin Institute, 327(6): 965-982. https://doi.org/10.1016/0016-0032(90)90072-Q

[5] Aziz, A. (1992). Optimum dimensions of extended surfaces operating in a convective environment. Applied Mechanics Reviews, 45(5): 155-173. https://doi.org/10.1115/1.3119754

[6] Aziz, A. (1985). Optimization of rectangular and triangular fins with convective boundary condition. International Communications in Heat and Mass Transfer, 12: 479-482. https://doi.org/10.1016/07351933(85)90041-7

[7] Aziz, A. (1994). Optimum design of a rectangular fin with a step change in cross-sectional area. International Communications in Heat and Mass Transfer, 21(3): 389401. https://doi.org/10.1016/0735-1933(94)90007-8

[8] Yu, L.T., Chen, C.O.K. (1998). Application of Taylor transformation to optimize rectangular fins with variable thermal parameters. Applied Mathematical Modelling, 22(1-2): 11-21. https://doi.org/10.1016/S0307904X(98)00004-3

[9] Poozesh, S., Nabi, S., Saber, M., Dinarvand, S., Fani, B. (2013). The efficiency of convective-radiative fin with temperature-dependent thermal conductivity by the differential transformation method. Research Journal of Applied Sciences, Engineering and Technology, 6(8): 1354-1359. http://dx.doi.org/10.19026/rjaset.6.3956

[10] Kang, H.S., Look Jr, D.C. (2007). Optimization of a thermally asymmetric convective and radiating annular fin. Heat Transfer Engineering, 28(4): 310-320. https://doi.org/10.1080/01457630601122609

[11] Kang, H.S., Look Jr, D.C. (2009). Optimization of a trapezoidal profile annular fin. Heat Transfer Engineering, 30(5): 359-367. https://doi.org/10.1080/01457630802414599

[12] Jasim, H.H., Söylemez, M.S. (2017). Optimization of a rectangular pin fin using rectangular perforations with different inclination angles. International Journal of Heat and Technology, 35(4): 969-977. https://doi.org/10.18280/ijht.350433

[13] Adhikari, R.C., Wood, D.H., Pahlevani, M. (2020). Optimizing rectangular fins for natural convection cooling using CFD. Thermal Science and Engineering Progress, 17: 100484. https://doi.org/10.1016/j.tsep.2020.100484

[14] Yu, C., Xue, X., Shi, K., Wang, R., Zhang, L., Shao, M. (2021). Optimization of wavy fin-and-elliptical tube heat exchanger using CFD, multi-objective genetic algorithm and radical basis function. Energy Science \& Engineering, $\quad 9(9)$ : $\quad$ 1359-1372. https://doi.org/10.1002/ese3.897

[15] Nguyen, Q., Yang, C.Y. (2016). Design of a longitudinal cooling fin with minimum volume by a modified Newton-Raphson method. Applied Thermal Engineering, $\quad$ 98: 169-178. https://doi.org/10.1016/j.applthermaleng.2015.12.035

[16] Quan, N., Son, N.H., Tuan, N.Q. (2018). Minimum volume of the longitudinal fin with rectangular and triangular profiles by a modified Newton-Raphson method. International Journal of Computational Methods, 15(5):

1850034. https://doi.org/10.1142/S0219876218500342

[17] Baskharone, E.A. (2013). The Finite Element Method with Heat Transfer and Fluid Mechanics Applications. Cambridge University Press. https://doi.org/10.1017/CBO9781139626668

[18] Beck, J.V., Blackwell, B., Clair Jr, C.R.S. (1985). Inverse Heat Conduction: Ill-Posed Problems. Wiley \& Sons. https://doi.org/10.1002/zamm.19870670331 


\section{NOMENCLATURE}

$k \quad$ the thermal conductivity, $\mathrm{W} \cdot \mathrm{m}^{-1} \cdot \mathrm{K}^{-1}$

$A_{b} \quad$ the cross-sectional area at the fin base, $\mathrm{m}^{2}$

$q_{\text {in }} \quad$ the total of toward the inside heat loss at the base, W.m. ${ }^{-2}$

$h$ the convective heat transfer coefficient, W. $\mathrm{m}^{-}$ ${ }^{2} . \mathrm{K}^{-1}$

n the the convective surface exterior normal vector

$L, l \quad$ the fin length, $\mathrm{m}$

$W, w \quad$ the fin width, $\mathrm{m}$

$H \quad$ the fin height, $\mathrm{m}$

$N$ the calculated temperature point number at the fin base

$V_{e} \quad$ the expected fin volume, $\mathrm{m}^{3}$

$V_{c} \quad$ the computed volume of the fin, $\mathrm{m}^{3}$

$V \quad$ the min volume, $\mathrm{m}^{3}$

$\mathbf{x}=\{w, l\} \quad$ the vector including the width variable of $w$ and the length variable of $l$

D the sensitivity matrix

\section{Greek symbols}

$\theta$

the undefined temperature field in the fin domain, $\mathrm{K}$ $\theta_{\infty}$ and the ambient and surrounding temperature, $\mathrm{K}$

$\theta_{\text {sur }}$

$\theta_{e} \quad$ the expected base temperature, $\mathrm{K}$

$\theta_{c}^{i} \quad$ the computed temperature, $\mathrm{K}$

$\boldsymbol{\Omega} \quad$ the diagonal matrix

$\mu \quad$ the parameter to control the iterative convergence

$e$ and $\delta \quad$ the convergence tolerances

\section{Subscripts}

$k \quad$ the iteration index

$b \quad$ base

in inward

e expected

c computed

$i \quad i$-th point/location

$\infty$ ambient

sur surrounding 